\title{
Multi-Jet Event Rates in Deep Inelastic Scattering and Determination of the Strong Coupling Constant
}

\author{
H1 Collaboration
}

\begin{abstract}
:
Jet event rates in deep inelastic ep scattering at HERA are investigated applying the modified JADE jet algorithm. The data are corrected for detector and hadronization effects and then compared with perturbative QCD predictions using next-toleading order calculations. The strong coupling constant $\alpha_{s}\left(M_{Z}^{2}\right)$ is determined evaluating the jet event rates. Values of $\alpha_{s}\left(Q^{2}\right)$ are extracted in four different bins of the negative squared momentum transfer $Q^{2}$ in the range from $40 \mathrm{GeV}^{2}$ to $4000 \mathrm{GeV}^{2}$. A combined fit of the renormalization group equation to these several $\alpha_{s}\left(Q^{2}\right)$ values results in $\alpha_{s}\left(M_{Z}^{2}\right)=0.117 \pm 0.003(\text { stat })_{-0.013}^{+0.009}($ sys $)+0.006($ jet algorithm $)$.
\end{abstract}


C. Adloff ${ }^{34}$, M. Anderson ${ }^{22}$, V. Andreev ${ }^{25}$, B. Andrieu ${ }^{28}$, V. Arkadov ${ }^{35}$, C. Arndt ${ }^{11}$, I. Ayyaz ${ }^{29}$, A. Babaev ${ }^{24}$, J. Bähr ${ }^{35}$, J. Bán ${ }^{17}$, P. Baranov ${ }^{25}$, E. Barrelet ${ }^{29}$, W. Bartel ${ }^{11}$, U. Bassler ${ }^{29}$, P. Bate ${ }^{22}$, M. Beck ${ }^{13}$, A. Beglarian ${ }^{11,40}$, O. Behnke ${ }^{11}$, H.-J. Behrend ${ }^{11}$, C. Beier ${ }^{15}$, A. Belousov ${ }^{25}$, Ch. Berger ${ }^{1}$, G. Bernardi ${ }^{29}$, G. Bertrand-Coremans ${ }^{4}$, P. Biddulph ${ }^{22}$, J.C. Bizot ${ }^{27}$, V. Boudry ${ }^{28}$, W. Braunschweig ${ }^{1}$, V. Brisson ${ }^{27}$, D.P. Brown ${ }^{22}$, W. Brückner ${ }^{13}$,

P. Bruel ${ }^{28}$, D. Bruncko ${ }^{17}$, J. Bürger ${ }^{11}$, F.W. Büsser ${ }^{12}$, A. Buniatian ${ }^{32}$, S. Burke ${ }^{18}$, G. Buschhorn ${ }^{26}$, D. Calvet ${ }^{23}$, A.J. Campbell ${ }^{11}$, T. Carli $^{26}$, E. Chabert ${ }^{23}$, M. Charlet ${ }^{4}$, D. Clarke $^{5}$, B. Clerbaux ${ }^{4}$, S. Cocks ${ }^{19}$, J.G. Contreras ${ }^{8}$, C. Cormack ${ }^{19}$, J.A. Coughlan ${ }^{5}$, M.-C. Cousinou ${ }^{23}$, B.E. Cox ${ }^{22}$, G. Cozzika ${ }^{10}$, J. Cvach $^{30}$, J.B. Dainton ${ }^{19}$, W.D. Dau ${ }^{16}$, K. Daum ${ }^{39}$, M. David ${ }^{10}$, M. Davidsson ${ }^{21}$, A. De Roeck ${ }^{11}$, E.A. De Wolf ${ }^{4}$, B. Delcourt ${ }^{27}$, C. Diaconu ${ }^{23}$, M. Dirkmann ${ }^{8}$, P. Dixon ${ }^{20}$, W. Dlugosz ${ }^{7}$, K.T. Donovan ${ }^{20}$, J.D. Dowell ${ }^{3}$, A. Droutskoi ${ }^{24}$, J. Ebert ${ }^{34}$, G. Eckerlin ${ }^{11}$, D. Eckstein ${ }^{35}$, V. Efremenko ${ }^{24}$, S. Egli ${ }^{37}$, R. Eichler ${ }^{36}$, F. Eisele ${ }^{14}$, E. Eisenhandler ${ }^{20}$, M. Enzenberger ${ }^{26}$, M. Erdmann ${ }^{14}$, A.B. Fahr ${ }^{12}$, L. Favart ${ }^{4}$, A. Fedotov ${ }^{24}$, R. Felst ${ }^{11}$, J. Feltesse ${ }^{10}$, J. Ferencei ${ }^{17}$, F. Ferrarotto ${ }^{32}$, M. Fleischer ${ }^{8}$, G. Flügge ${ }^{2}$, A. Fomenko ${ }^{25}$, J. Formánek ${ }^{31}$, J.M. Foster ${ }^{22}$, G. Franke ${ }^{11}$, E. Gabathuler ${ }^{19}$, K. Gabathuler ${ }^{33}$, F. Gaede ${ }^{26}$, J. Garvey ${ }^{3}$, J. Gayler ${ }^{11}$, R. Gerhards ${ }^{11}$, S. Ghazaryan ${ }^{11,40}$, A. Glazov ${ }^{35}$, L. Goerlich ${ }^{6}$, N. Gogitidze ${ }^{25}$, M. Goldberg ${ }^{29}$, I. Gorelov ${ }^{24}$, C. Grab ${ }^{36}$, H. Grässler ${ }^{2}$, T. Greenshaw ${ }^{19}$, R.K. Griffiths ${ }^{20}$, G. Grindhammer ${ }^{26}$, T. Hadig ${ }^{1}$, D. Haidt ${ }^{11}$, L. Hajduk ${ }^{6}$, T. Haller ${ }^{13}$, M. Hampel ${ }^{1}$, V. Haustein ${ }^{34}$, W.J. Haynes ${ }^{5}$, B. Heinemann ${ }^{11}$, G. Heinzelmann ${ }^{12}$, R.C.W. Henderson ${ }^{18}$, S. Hengstmann ${ }^{37}$, H. Henschel ${ }^{35}$, R. Heremans ${ }^{4}$, I. Herynek ${ }^{30}$, K. Hewitt ${ }^{3}$, K.H. Hiller ${ }^{35}$, C.D. Hilton ${ }^{22}$, J. Hladký ${ }^{30}$, D. Hoffmann ${ }^{11}$, T. Holtom ${ }^{19}$, R. Horisberger ${ }^{33}$, V.L. Hudgson ${ }^{3}$, S. Hurling ${ }^{11}$, M. Ibbotson ${ }^{22}$, Ç. İşsever ${ }^{8}$, H. Itterbeck ${ }^{1}$, M. Jacquet ${ }^{27}$, M. Jaffre ${ }^{27}$, D.M. Jansen ${ }^{13}$, L. Jönsson ${ }^{21}$, D.P. Johnson ${ }^{4}$, H. Jung ${ }^{21}$, H.K. Kästlii ${ }^{36}$, M. Kander ${ }^{11}$, D. Kant ${ }^{20}$, M. Kapichine ${ }^{9}$, M. Karlsson ${ }^{21}$, O. Karschnik ${ }^{12}$, J. Katzy ${ }^{11}$, O. Kaufmann ${ }^{14}$, M. Kausch ${ }^{11}$, I.R. Kenyon ${ }^{3}$, S. Kermiche ${ }^{23}$, C. Keuker ${ }^{1}$, C. Kiesling ${ }^{26}$, M. Klein ${ }^{35}$, C. Kleinwort ${ }^{11}$, G. Knies ${ }^{11}$, J.H. Köhne ${ }^{26}$, H. Kolanoski ${ }^{38}$, S.D. Kolya ${ }^{22}$, V. Korbel ${ }^{11}$, P. Kostka ${ }^{35}$, S.K. Kotelnikov ${ }^{25}$,

T. Krämerkämper ${ }^{8}$, M.W. Krasny ${ }^{29}$, H. Krehbiel ${ }^{11}$, D. Krücker ${ }^{26}$, K. Krüger ${ }^{11}$, A. Küpper ${ }^{34}$, H. Küster ${ }^{21}$, M. Kuhlen ${ }^{26}$, T. Kurča ${ }^{35}$, B. Laforge ${ }^{10}$, R. Lahmann ${ }^{11}$, M.P.J. Landon ${ }^{20}$, W. Lange ${ }^{35}$, U. Langenegger ${ }^{36}$, A. Lebedev ${ }^{25}$, F. Lehner ${ }^{11}$, V. Lemaitre ${ }^{11}$, S. Levonian ${ }^{11}$, M. Lindstroem ${ }^{21}$, B. List ${ }^{11}$, G. Lobo ${ }^{27}$, V. Lubimov ${ }^{24}$, D. Lüke ${ }^{8,11}$, L. Lytkin ${ }^{13}$, N. Magnussen ${ }^{34}$, H. Mahlke-Krüger ${ }^{11}$, E. Malinovski ${ }^{25}$, R. Maraček ${ }^{17}$, P. Marage ${ }^{4}$, J. Marks ${ }^{14}$, R. Marshall ${ }^{22}$, G. Martin ${ }^{12}$, H.-U. Martyn ${ }^{1}$, J. Martyniak ${ }^{6}$, S.J. Maxfield ${ }^{19}$, S.J. McMahon ${ }^{19}$, T.R. McMahon ${ }^{19}$, A. Mehta ${ }^{5}$, K. Meier ${ }^{15}$, P. Merkel $^{11}$, F. Metlica ${ }^{13}$, A. Meyer ${ }^{12}$, A. Meyer ${ }^{11}$, H. Meyer ${ }^{34}$, J. Meyer ${ }^{11}$, P.-O. Meyer ${ }^{2}$, S. Mikocki ${ }^{6}$, D. Milstead ${ }^{11}$, J. Moeck ${ }^{26}$, R. Mohr ${ }^{26}$, S. Mohrdieck ${ }^{12}$, F. Moreau ${ }^{28}$, J.V. Morris ${ }^{5}$, E. Mroczko ${ }^{6}$, D. Müller ${ }^{37}$, K. Müller ${ }^{11}$, P. Murín ${ }^{17}$, V. Nagovizin ${ }^{24}$, B. Naroska ${ }^{12}$, Th. Naumann ${ }^{35}$, I. Négri2 ${ }^{23}$, P.R. Newman ${ }^{3}$, H.K. Nguyen ${ }^{29}$, T.C. Nicholls ${ }^{11}$, F. Niebergall ${ }^{12}$, C. Niebuhr ${ }^{11}$, Ch. Niedzballa ${ }^{1}$, H. Niggli ${ }^{36}$, D. Nikitin ${ }^{9}$, O. $\mathrm{Nix}^{15}$, G. Nowak ${ }^{6}$, T. Nunnemann ${ }^{13}$, H. Oberlack ${ }^{26}$, J.E. Olsson ${ }^{11}$, D. Ozerov ${ }^{24}$, P. Palmen ${ }^{2}$, V. Panassik ${ }^{9}$, C. Pascaud ${ }^{27}$, S. Passaggio ${ }^{36}$, G.D. Patel ${ }^{19}$, H. Pawletta ${ }^{2}$, E. Perez ${ }^{10}$, J.P. Phillips ${ }^{19}$, A. Pieuchot ${ }^{11}$, D. Pitzl ${ }^{36}$, R. Pöschl ${ }^{8}$, G. Pope ${ }^{7}$, B. Povh ${ }^{13}$, K. Rabbertz ${ }^{1}$, J. Rauschenberger ${ }^{12}$, P. Reimer ${ }^{30}$, B. Reisert ${ }^{26}$, H. Rick ${ }^{11}$, S. Riess ${ }^{12}$, E. Rizvi ${ }^{11}$, P. Robmann ${ }^{37}$, R. Roosen ${ }^{4}$, K. Rosenbauer ${ }^{1}$, A. Rostovtsev ${ }^{24,12}$, F. Rouse ${ }^{7}$, C. Royon $^{10}$, S. Rusakov ${ }^{25}$, K. Rybicki ${ }^{6}$, D.P.C. Sankey ${ }^{5}$, P. Schacht ${ }^{26}$, J. Scheins ${ }^{1}$, S. Schleif ${ }^{15}$, P. Schleper ${ }^{14}$, D. Schmidt ${ }^{11}$, D. Schmidt ${ }^{34}$, L. Schoeffel ${ }^{10}$, V. Schröder ${ }^{11}$, H.-C. Schultz-Coulon ${ }^{11}$, B. Schwab ${ }^{14}$, F. Sefkow ${ }^{37}$, A. Semenov ${ }^{24}$, V. Shekelyan ${ }^{26}$, I. Sheviakov ${ }^{25}$, L.N. Shtarkov ${ }^{25}$, G. Siegmon ${ }^{16}$, Y. $\operatorname{Sirois}^{28}$, T. Sloan ${ }^{18}$, P. Smirnov ${ }^{25}$, M. Smith ${ }^{19}$, V. Solochenko ${ }^{24}$, Y. Soloviev ${ }^{25}$, V .Spaskov ${ }^{9}$, A. Specka ${ }^{28}$, J. Spiekermann ${ }^{8}$, H. Spitzer ${ }^{12}$, F. Squinabol ${ }^{27}$, P. Steffen ${ }^{11}$, R. Steinberg ${ }^{2}$, J. Steinhart ${ }^{12}$, B. Stella ${ }^{32}$, A. Stellberger ${ }^{15}$, J. Stiewe ${ }^{15}$, U. Straumann ${ }^{14}$, W. Struczinski ${ }^{2}$, J.P. Sutton ${ }^{3}$, M. Swart ${ }^{15}$, S. Tapprogge ${ }^{15}$, M. Taševský ${ }^{30}$, V. Tchernyshov ${ }^{24}$, S. Tchetchelnitski ${ }^{24}$, J. Theissen ${ }^{2}$, G. Thompson ${ }^{20}$, P.D. Thompson ${ }^{3}$, N. Tobien ${ }^{11}$, R. Todenhagen ${ }^{13}$, P. Truöl ${ }^{37}$, G. Tsipolitis ${ }^{36}$, 
J. Turnau ${ }^{6}$, E. Tzamariudakii ${ }^{11}$, S. Udluft ${ }^{26}$, A. Usik ${ }^{25}$, S. Valkár ${ }^{31}$, A. Valkárováá ${ }^{31}$, C. Vallée ${ }^{23}$, P. Van $\operatorname{Esch}^{4}$, A. Van Haecke ${ }^{10}$, P. Van Mechelen ${ }^{4}$, Y. Vazdik ${ }^{25}$, G. Villet ${ }^{10}$, K. Wacker ${ }^{8}$, R. Wallny ${ }^{14}$, T. Walter ${ }^{37}$, B. Waugh ${ }^{22}$, G. Weber ${ }^{12}$, M. Weber ${ }^{15}$, D. Wegener ${ }^{8}$, A. Wegner ${ }^{26}$, T. Wengler ${ }^{14}$, M. Werner ${ }^{14}$, L.R. West ${ }^{3}$, S. Wiesand ${ }^{34}$, T. Wilksen ${ }^{11}$, S. Willard ${ }^{7}$, M. Winde ${ }^{35}$, G.-G. Winter ${ }^{11}$, C. Wittek ${ }^{12}$, E. Wittmann ${ }^{13}$, M. Wobisch $^{2}$, H. Wollatz ${ }^{11}$, E. Wünsch ${ }^{11}$, J. Žáček ${ }^{31}$, J. Zálešák ${ }^{31}$, Z. Zhang ${ }^{27}$, A. Zhokin ${ }^{24}$, P. Zini ${ }^{29}$, F. Zomer ${ }^{27}$, J. Zsembery ${ }^{10}$ and M. zurNedden ${ }^{37}$

${ }^{1}$ I. Physikalisches Institut der RWTH, Aachen, Germany ${ }^{a}$

${ }^{2}$ III. Physikalisches Institut der RWTH, Aachen, Germany ${ }^{a}$

3 School of Physics and Space Research, University of Birmingham, Birmingham, UK ${ }^{b}$

${ }^{4}$ Inter-University Institute for High Energies ULB-VUB, Brussels; Universitaire Instelling Antwerpen, Wilrijk; Belgium ${ }^{c}$

${ }^{5}$ Rutherford Appleton Laboratory, Chilton, Didcot, $U K^{b}$

${ }^{6}$ Institute for Nuclear Physics, Cracow, Poland ${ }^{d}$

7 Physics Department and IIRPA, University of California, Davis, California, USA ${ }^{e}$

8 Institut für Physik, Universität Dortmund, Dortmund, Germany ${ }^{a}$

9 Joint Institute for Nuclear Research, Dubna, Russia

${ }^{10}$ DSM/DAPNIA, CEA/Saclay, Gif-sur-Yvette, France

11 DESY, Hamburg, Germany ${ }^{a}$

${ }^{12}$ II. Institut für Experimentalphysik, Universität Hamburg, Hamburg, Germany ${ }^{a}$

${ }^{13}$ Max-Planck-Institut für Kernphysik, Heidelberg, Germany ${ }^{a}$

${ }^{14}$ Physikalisches Institut, Universität Heidelberg, Heidelberg, Germany ${ }^{a}$

15 Institut für Hochenergiephysik, Universität Heidelberg, Heidelberg, Germany ${ }^{a}$

16 Institut für experimentelle und angewandte Physik, Universität Kiel, Kiel, Germany ${ }^{a}$

17 Institute of Experimental Physics, Slovak Academy of Sciences, Košice, Slovak Republic ${ }^{f, j}$

18 School of Physics and Chemistry, University of Lancaster, Lancaster, UK ${ }^{b}$

19 Department of Physics, University of Liverpool, Liverpool, $U K^{b}$

20 Queen Mary and Westfield College, London, UK

21 Physics Department, University of Lund, Lund, Sweden ${ }^{g}$

22 Department of Physics and Astronomy, University of Manchester, Manchester, UK ${ }^{b}$

${ }^{23}$ CPPM, Université d'Aix-Marseille II, IN2P3-CNRS, Marseille, France

${ }^{24}$ Institute for Theoretical and Experimental Physics, Moscow, Russia

${ }^{25}$ Lebedev Physical Institute, Moscow, Russiaf,k

${ }^{26}$ Max-Planck-Institut für Physik, München, Germany ${ }^{a}$

27 LAL, Université de Paris-Sud, IN2P3-CNRS, Orsay, France

${ }^{28}$ LPNHE, Ecole Polytechnique, IN2P3-CNRS, Palaiseau, France

29 LPNHE, Universités Paris VI and VII, IN2P3-CNRS, Paris, France

30 Institute of Physics, Academy of Sciences of the Czech Republic, Praha, Czech Republic ${ }^{f, h}$

31 Nuclear Center, Charles University, Praha, Czech Republic ${ }^{f, h}$

${ }^{32}$ INFN Roma 1 and Dipartimento di Fisica, Università Roma 3, Roma, Italy

33 Paul Scherrer Institut, Villigen, Switzerland

${ }^{34}$ Fachbereich Physik, Bergische Universität Gesamthochschule Wuppertal, Wuppertal, Germany ${ }^{a}$

${ }^{35}$ DESY, Institut für Hochenergiephysik, Zeuthen, Germany ${ }^{a}$

36 Institut für Teilchenphysik, ETH, Zürich, Switzerland ${ }^{i}$

37 Physik-Institut der Universität Zürich, Zürich, Switzerland ${ }^{i}$

38 Institut für Physik, Humboldt-Universität, Berlin, Germany ${ }^{a}$

39 Rechenzentrum, Bergische Universität Gesamthochschule Wuppertal, Wuppertal, Germany ${ }^{a}$

40 Vistor from Yerevan Physics Institute, Armenia

${ }^{a}$ Supported by the Bundesministerium für Bildung, Wissenschaft, Forschung und Technologie, 
FRG, under contract numbers 7AC17P, 7AC47P, 7DO55P, 7HH17I, 7HH27P, 7HD17P, 7HD27P, 7KI17I, 6MP17I and 7WT87P

${ }^{b}$ Supported by the UK Particle Physics and Astronomy Research Council, and formerly by the UK Science and Engineering Research Council

${ }^{c}$ Supported by FNRS-FWO, IISN-IIKW

$d$ Partially supported by the Polish State Committee for Scientific Research, grant no. 115/E-343/SPUB/P03/002/97 and grant no. 2P03B 05513

e Supported in part by US DOE grant DE F603 91ER40674

$f$ Supported by the Deutsche Forschungsgemeinschaft

$g$ Supported by the Swedish Natural Science Research Council

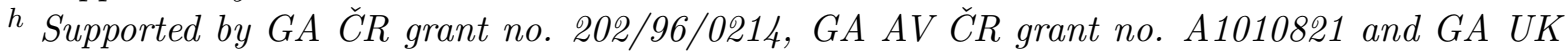
grant no. 177

${ }^{i}$ Supported by the Swiss National Science Foundation

j Supported by VEGA SR grant no. 2/5167/98

${ }^{k}$ Supported by Russian Foundation for Basic Research grant no. 96-02-00019 


\section{Introduction}

Multi-jet production in neutral current deep inelastic positron proton scattering (DIS) is investigated using data taken in 1994 and 1995 with the H1 detector at HERA at DESY, where $820 \mathrm{GeV}$ protons collide with $27.5 \mathrm{GeV}$ positrons. A measurement of the relative production rates of events with different jet multiplicities will lead to a better understanding of the underlying elementary processes sensitive to perturbative QCD (pQCD) including its basic parameter, the strong coupling constant.

The DIS reaction can be written as $e+p \rightarrow e+X$, where $X$ denotes an arbitrary hadronic final state. The process is sketched in figure 1, in which the four-momentum vectors of the particles are given in brackets to define the kinematic quantities used later in the text. The incident positron is scattered over a wide range of the negative squared four-momentum transfer $Q^{2}=$ $-q^{2}=-\left(k-k^{\prime}\right)^{2}$. The absorption of the virtual photon by the proton produces a hadronic final state with a squared invariant mass of $W^{2}=(q+P)^{2}$.

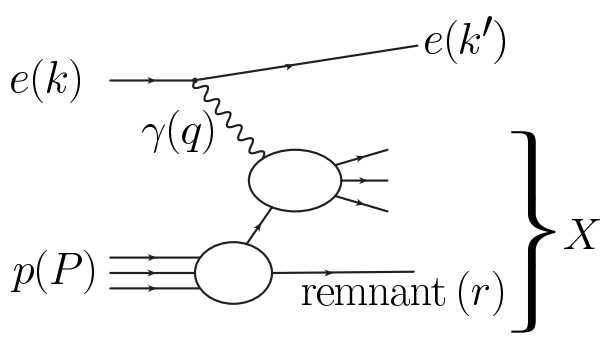

Figure 1: Sketch of neutral current (NC) deep inelastic ep scattering (DIS) reaction ep $\rightarrow e X$, where the four-momentum vectors are given in brackets.

In the range of $Q^{2}$ investigated in this paper, neutral current DIS reactions can be described as purely electromagnetic scattering of the positron off a quark via single photon exchang®. To lowest order, this leads to $(1+1)$ jet events. One jet is caused by the scattered quark, the other $(+1)$ by the remaining part of the proton, the proton remnant, which can only be partially observed in the $\mathrm{H} 1$ detector.

Higher jet multiplicities are described in the framework of perturbative QCD. The corresponding cross sections are expressed as power series in the strong coupling constant $\alpha_{s}$, such that the leading order (LO) contribution to $(2+1)$ jet events is of order $\mathcal{O}\left(\alpha_{s}\right)$. Two generic graphs contribute (figure 2), where the hard scattering process is either gluon (2a) or quark (2b) induced. The resulting jets from the hard scattering are called "current jets".

By applying the modified JADE jet algorithm [1, 2] to the data it has been shown in previous publications [3, 何, that jet production at HERA is dominated by the two diagrams of fig. 2 in a large region of phase space. This motivates a quantitative comparison to pQCD predictions including real and virtual corrections to the diagrams in question.

Up to now the theoretical calculations [5, 6] available for such comparisons neglected terms containing the product of $W^{2}$ and the JADE jet resolution parameter $y_{c u t}$ and thus were not valid for large values of $W^{2}$ accessed at low values of $Q^{2}$. In recent NLO QCD-calculations [7, 8] no such terms are neglected. Hence it is appropriate to compare more measurements with these new predictions in a larger region of phase space, especially for low $Q^{2}$ where higher statistics are available. Thus this analysis supersedes the previous H1 publication [3].

\footnotetext{
${ }^{1}$ In the $Q^{2}$ range under study $\left(Q^{2}<4000 \mathrm{GeV}^{2}\right.$ with the present statistics) effects due to the exchange of the intermediate vector boson $Z^{\circ}$ can be neglected.
} 


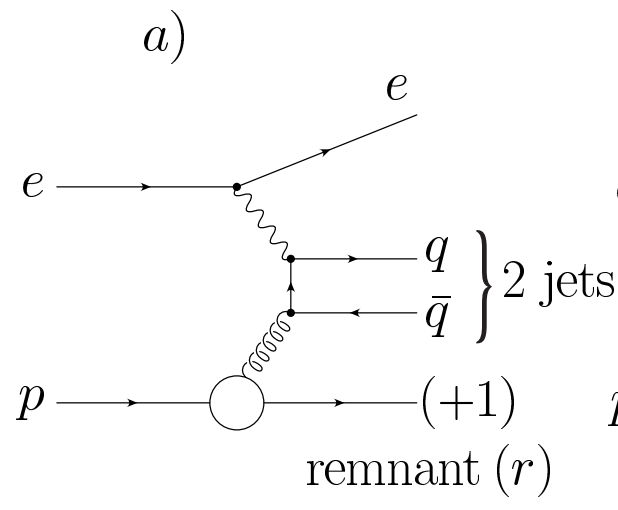

b)

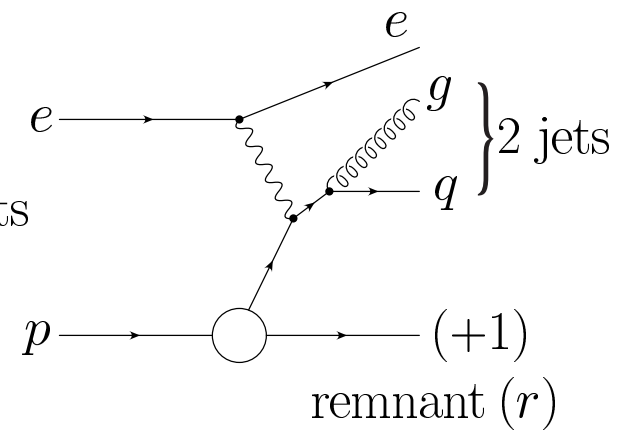

Figure 2: Generic diagrams of the QCD LO contributions to $(2+1)$ jet events in neutral current $D I S$.

A necessary prerequisite to the analysis reported here is to define a phase space region in which NLO calculations are able to describe the data after correcting for hadronization and detector effects; the dependence of the $(2+1)$ jet event rate on $Q^{2}$, together with other hadronic control variables, should be well predicted by the fixed order calculation.

The presented quantitative $\mathrm{pQCD}$ analysis is based on the previous $\mathrm{H} 1$ publication [3]. The measured $(2+1)$ jet event rates in different bins of $Q^{2}$ are corrected for detector and hadronization effects to the parton level. Taking the known parton densities in the proton, values of $\alpha_{s}$ are extracted as a function of $Q^{2}$ which fit best the corrected jet event rates. It is thus possible to investigate the scale dependence of the strong coupling constant using only one observable, the $(2+1)$ jet event rate, in a single experiment. Then $\alpha_{s}\left(M_{Z}^{2}\right)$ is determined by performing a fit of the QCD parameter $\Lambda$ in the renormalization group equation to the independent $\alpha_{s}\left(Q^{2}\right)$ values. Here $M_{Z}$ is the mass of the neutral vector boson $Z^{0}$. The use of a recent NLO QCD calculation program allows a study of the dependence of the obtained $\alpha_{s}\left(M_{Z}^{2}\right)$ on the choice of the jet algorithm.

\section{$2 \quad$ H1 Apparatus}

A detailed discussion of the H1 apparatus can be found elsewhere [9]. Here emphasis is put on describing the main features of those detector relevant to this analysis, which makes use mainly of the calorimeters and, to a lesser extent, of the central and backward tracking systems. The polar angle, $\theta$, is defined with respect to the proton beam direction, which is identified with the $+z$-axis.

The inner part of the detector consists of the central tracking chamber supplemented by a forward tracking detector and a backward proportional chamber (BPC), covering the polar angular ranges $25^{\circ}<\theta<155^{\circ}, 7^{\circ}<\theta<25^{\circ}$ and $155^{\circ}<\theta<175^{\circ}$, respectively. The central and forward tracking devices are used to determine the vertex position. The central tracking chamber and the BPC, together with the vertex, are used to measure the positron scattering angle in the central and backward region. The angular resolution achieved is better than 1 mrad.

The energy of the scattered positron is measured by the liquid argon (LAr) calorimeter and in a backward electromagnetic lead-scintillator calorimeter (BEMC); the LAr calorimeter is also used for a measurement of the hadronic energy flow. A superconducting solenoid outside the LAr calorimeter provides a uniform magnetic field of $1.15 \mathrm{~T}$ parallel to the proton beam axis throughout the tracking region. 
The LAr calorimeter [10] extends over the polar angular range $4^{\circ}<\theta<154^{\circ}$ with full azimuthal coverage. The scattered positron enters the LAr for events in which $Q^{2}$ is larger than about $100 \mathrm{GeV}^{2}$. The calorimeter consists of an electromagnetic section with lead absorbers, corresponding to a depth between 20 and 30 radiation lengths, and a hadronic section with steel absorbers. The total depth of the LAr calorimeter varies between 4.5 and 8 hadronic interaction lengths. Test beam measurements of LAr calorimeter modules have demonstrated energy resolutions of $\sigma(E) / E \approx 0.12 / \sqrt{E / \mathrm{GeV}} \oplus 0.01$ for positrons [11] and $\sigma(E) / E \approx 0.5 / \sqrt{E / \mathrm{GeV}} \oplus 0.02$ for charged pions [12]. The uncertainty of the absolute energy scale for positrons is $3 \%$ determined with studies based on the double angle method of kinematic reconstruction [13]. The hadronic energy scale has been verified from the balance of transverse momentum between the hadronic final state and the scattered positron in DIS events and is known to a precision of $4 \%$ [14].

The BEMC [15], with a thickness of 21.7 radiation lengths, covers the backward region of the detector, $151^{\circ}<\theta<176^{\circ}$. It is mainly used to measure positrons from DIS processes at low $Q^{2}$. The acceptance region corresponds to $Q^{2}$ values in the approximate range $5 \leq Q^{2} \leq 100$ $\mathrm{GeV}^{2}$. A resolution of $\sigma(E) / E \approx 0.10 / \sqrt{E / \mathrm{GeV}} \oplus 0.02$ has been achieved. By adjusting the measured positron energy spectrum to the kinematic peak the BEMC energy scale is known to an accuracy of 1\% 15]. Since 1995 the BEMC and BPC in the backward region have been replaced by a lead/scintillating fibre calorimeter (SPACAL) [16] and a drift chamber (BDC) [17].

\section{Event Selection}

The data are divided into two sub-samples depending on the location of the detected positron in the BEMC or in the LAr calorimeter. The scattered positron is defined as either the most energetic cluster detected in the BEMC or a contiguous cluster in the LAr whose energy in the electromagnetic section exceeds $80 \%$ of the total cluster energy. The used data samples correspond to integrated luminosities of $2.8 \mathrm{pb}^{-1}$ taken in 1994 for $Q^{2}<100 \mathrm{GeV}^{2}$ (positrons in BEMC) and of $7.1 \mathrm{pb}^{-1}$ taken in 1994 and 1995 for $Q^{2}>100 \mathrm{GeV}^{2}$ (positron in LAr calorimeter). All further selection criteria follow closely those used in the H1 measurement of the proton structure function $F_{2}$ [18.

Events in the first sub-sample (BEMC) must satisfy the following requirements:

1. The four-momentum transfer $Q^{2}$ must be between 10 and $100 \mathrm{GeV}^{2}$.

2. The energy $E_{e}^{\prime}$ of the scattered positron must be greater than $14 \mathrm{GeV}$, corresponding to a fractional positron energy loss in the proton rest-frame of $y=q P / k P \lesssim 0.5$ (see fig. 11 for the meaning of four-momentum vectors), thus eliminating background from photoproduction, where a hadron is misidentified as a positron, to a negligible level $(<1 \%)$. In addition this cut suppresses radiative DIS events.

3. The angle of the scattered positron must be in the range $160^{\circ}<\theta_{e}<173^{\circ}$ to ensure that it is well contained in the BEMC.

Events in the second sub-sample (LAr) must satisfy the following requirements:

1. $Q^{2}$ must be greater than $100 \mathrm{GeV}^{2}$.

2. The energy of the scattered positron $E_{e}^{\prime}$ must be greater than $11 \mathrm{GeV}, y$ less than 0.7 , and the quantity $\delta=\sum_{\text {clusters }}\left(E-P_{z}\right.$ ) (with $E$ and $P_{z}$ being the energy and longitudinal momentum of the calorimetric energy clusters) is required to be within $38 \mathrm{GeV}<\delta<70$ $\mathrm{GeV}$. These cuts exclude efficiently photo-production events $(<1 \%)$, in which the scattered positron is lost down the beam pipe. As for the cut in $y$ discussed above, the lower $\delta$-cut further reduces radiative DIS events. 
3. The angle $\theta_{e}$ should fulfill $10^{\circ}<\theta_{e}<150^{\circ}$ to ensure that the positron is contained in the LAr calorimeter and to avoid the transition region between the LAr and the BEMC.

Two additional requirements were imposed on both sub-samples:

1. An event vertex from charged tracks within $\pm 30 \mathrm{~cm}$ around the maximum, $z_{\text {mean }}$, of the vertex distribution is required; $z_{\text {mean }}$ was located at $z=+5 \mathrm{~cm}(+1 \mathrm{~cm})$ in 1994 (1995).

2. $W^{2}>5000 \mathrm{GeV}^{2}$, where $W^{2}$ is calculated using the double angle method. This method relies on the angle of the scattered positron and an inclusive angle of the hadronic final state, and is independent of the jet classification. The $W^{2}$ cut ensures enough hadronic activity in the detector and is equivalent to a cut on the lepton variable $y(y>0.055)$.

In both samples $Q^{2}$ and $y$ are calculated from the energy and angle of the scattered positron. The selected event sample contains 112985 events with $Q^{2}<100 \mathrm{GeV}^{2}$ and 5410 (8280) events with $100 \mathrm{GeV}^{2}<Q^{2}<4000 \mathrm{GeV}^{2}$ selected from the data taken in 1994 (1995).

\section{Jet Reconstruction}

All events are subject to a jet classification using the energy clusters in the LAr calorimeter always excluding the scattered positron. In order to compare the measurement to QCD predictions the same jet classification is applied to Monte-Carlo events after passing the full H1 detector simulation and after using the same reconstruction algorithm as for the data. The influence of hadronization and detector effects on the number of reconstructed jets at this "detector level" is studied by determining in addition the jets at the parton and the hadron level. At the "parton level" the coloured partons generated according to pQCD calculations will form the jets, whereas at the "hadron level", the fully generated hadronic final state is analysed.

The analysis presented here is based on a modified JADE jet algorithm [1]. In order to take into account the invisible part of the proton remnant $r$ the modified JADE jet algorithm includes a pseudo-particle in the clustering procedure representing the missing longitudinal momentum in the event [2]. The JADE algorithm is applied in the laboratory frame. Two objects $i, j$ with a squared mass $m_{i j}^{2}$ are combined to one new object if $y_{i j}=m_{i j}^{2} / M_{r e f}^{2}<y_{c u t}$, where $y_{c u t}$ is the resolution parameter. The scale $M_{\text {ref }}$ is taken to be the invariant mass $W$ of the hadronic system $X$, where $W$ is calculated as the invariant mass of the four-vector sum of all hadronic objects. From studies of the resolution parameter [19, 20], $y_{\text {cut }}$ is set to 0.02. This choice ensures satisfactory correlations between jets reconstructed at the parton and the detector level, and high statistics of $(2+1)$ jet events, keeping the contribution of $(3+1)$ jet events below $4 \%$.

Besides the modified JADE jet algorithm two of its variants, the E0 and the P algorithm, are investigated, which differ in the combination of two objects $i$ and $j$ to a new object $k$. For the $\mathrm{JADE}$ algorithm the new four-momentum vector $p_{k}$ is given by $p_{k}=p_{i}+p_{j}$, whereas for the E0

and $\mathrm{P}$ algorithm the new four-momenta are massless and given by $p_{k}=\left[E_{i}+E_{j},\left(E_{i}+E_{j}\right) \cdot \frac{\vec{p}_{i}+\vec{p}_{j}}{\mid \vec{p}_{i}+\vec{p}_{j}}\right]$ and $p_{k}=\left[\left|\vec{p}_{i}+\vec{p}_{j}\right|, \vec{p}_{i}+\vec{p}_{j}\right]$, respectively. The resolution criterion for all of these algorithms is given by $y_{i j}=2 E_{i} E_{j}\left(1-\cos \theta_{i j}\right) / W^{2}$, where $E_{i}, E_{j}$ denote the energies and $\theta_{i j}$ is the angle between the two objects $i, j$ under consideration. As long as there is no restriction on the momenta of the resulting jets, the JADE and E0 algorithm are equivalent in the theoretical NLO $(2+1)$ jet cross section calculations. For these two algorithms, only the $(1+1)$ jet cross section is affected differently by cuts on the current jet angles. The difference, which is marginal, is caused by events where the clustering of all three partons and the proton remnant leads to a $(1+1)$ jet event. 


\section{Outline of the QCD Analysis}

In order to study the applicability of pQCD quantitatively, the $(2+1)$ jet event rate for different bins of $Q^{2}$ (see table 1) is chosen [3, 19] to determine $\alpha_{s}\left(\mu_{r}^{2}\right)$ as a function of the renormalization scale $\mu_{r}^{2}$, which is taken to be $Q^{2}$. The NLO QCD calculation can be used to calculate an expression for $R_{2+1}\left(Q^{2}, y_{\text {cut }}\right)$ in terms of a power series in $\alpha_{s}\left(Q^{2}\right)$ with coefficients $a\left(Q^{2}, y_{\text {cut }}\right)$ and $b\left(Q^{2}, y_{\text {cut }}\right)$ :

$$
R_{2+1}\left(Q^{2}, y_{\text {cut }}\right)=\frac{\sigma_{2+1}\left(Q^{2}, y_{\text {cut }}\right)}{\sigma_{\text {norm }}\left(Q^{2}, y_{\text {cut }}\right)}=a\left(Q^{2}, y_{\text {cut }}\right) \alpha_{s}\left(Q^{2}\right)+b\left(Q^{2}, y_{\text {cut }}\right) \alpha_{s}^{2}\left(Q^{2}\right)+\mathcal{O}\left(\alpha_{s}^{3}\right) .
$$

Taking the variation of $\alpha_{s}$ with $Q^{2}$ to be negligible over each bin of $Q^{2}$, to $\mathcal{O}\left(\alpha_{s}^{2}\right)$ this equation reduces to a quadratic form for $\alpha_{s}$ with coefficients determined from the integrals of $a\left(Q^{2}, y_{\text {cut }}\right)$ and $b\left(Q^{2}, y_{\text {cut }}\right)$ over each $Q^{2}$ bin. This quadratic expression can then be solved for $\alpha_{s}$ using the measured jet event rates for different $Q^{2}$.

The cross section used for normalization, $\sigma_{\text {norm }}\left(Q^{2}\right)$, is taken as the sum of jet cross sections, where one or two current jets $\left(\sigma_{1+1}\right.$ for $(1+1)$ and $\sigma_{2+1}$ for $(2+1)$ events) are found in the acceptance region. Experimentally $R_{2+1}$ is obtained as $N_{2+1} /\left(N_{2+1}+N_{1+1}\right)$, where $N_{i+1}$ denotes the number of events with $i$ observed current jets.

The theoretical $\mathcal{O}\left(\alpha_{s}^{2}\right)$ Monte Carlo integration programs, MEPJET [7] and DISENT [8], used for the prediction of jet cross sections are only available at the parton level and not as event generators. Both programs give the same results to within $1 \%$ precision. Consequently the relation between observed jets in the detector and the underlying partonic jet configuration has to be obtained in form of correction factors from a LO event generator which includes a simulation of higher-order effects and hadronization.

The MEPJET Monte Carlo program is used to compare the NLO prediction with the measured jet event rates and distributions of kinematic variables. It is necessary to apply to the NLO Monte Carlo the same phase space restrictions in terms of positron variables and jet quantities as is done in the experimental event selection. The forward direction is the most crucial region for the comparison. The emission of multi-gluon radiation from initial state partons entering the hard scattering process causes a higher reconstructed jet multiplicity. The influence of multigluon radiation increases with decreasing $Q^{2}$ because at low $Q^{2}$ very low values of the scaling variable $x_{B}=Q^{2} / 2 q P$ are reached, which increases the permitted phase space $\left(\xi>x_{B}\right)$ for the momentum fraction $\xi$ of the proton carried by the parton initiating the multi-gluon radiation. This initial state radiation is naturally not taken into account by a fixed order theory (as in MEPJET). As a consequence the jet event rate which is predicted by the NLO calculations is much smaller than the measured event rate without further cuts, particularly at low values of $Q^{2}$. Therefore the event generator Monte Carlo is not only used to calculate the correction factors but also to determine cuts necessary to suppress the influence of multi-gluon radiation and hadronization effects.

To define such a phase space region where the NLO QCD prediction can describe the partonic scattering underlying the data events, an event generator is necessary, which has a parton level comparable to the partonic configuration of the NLO QCD calculation and describes the data. Therefore the acceptance region for the current jets is chosen using the following criteria:

- The dependence of the jet event rate on $Q^{2}$ predicted by the NLO calculation should be in good agreement with the prediction of the event generator at the parton level. For a reasonable range of the QCD parameter $\Lambda \frac{(4)}{M S}$ as used in MEPJET ${ }^{3}\left(150<\Lambda \frac{(4)}{M S}<400 \mathrm{MeV}\right)$

\footnotetext{
${ }^{2}$ The factorization scale $\mu_{f}^{2}$ is also set to $Q^{2}$.
}

${ }^{3}$ In MEPJET the effective number of flavors $n_{f}$ contributing to the QCD parameter $\Lambda_{\overline{M S}}^{\left(n_{f}\right)}$ (the calculation assumes zero quark masses) is fixed to 5 ; but in the text presented here always the corresponding $\Lambda_{\frac{(4)}{M S}}$ will be given. 
also the absolute values of the jet event rate should be close to the predicted values from the event generator in each bin of $Q^{2}$. In addition other reconstructed jet variable distributions at partonic level, such as jet angles, should be in acceptable agreement with the NLO calculation.

- The measured $(2+1)$ jet event rates and the other data distributions of the chosen jet variables should be described by the event generator model at the detector level.

It is assumed that if these requirements are fulfilled the correction factors from measured jets to the NLO parton configuration can be provided by the event generator.

As event generator the program LEPTO 6.5 [21] is used. LEPTO enables event generation by matching leading log parton showers to the LO matrix elements (MEPS). In the event generator a momentum scale $Q_{0}$ specifies the termination of the parton shower, that is the boundary between the perturbative and non-perturbative stages of jet development. For this analysis, this scale is taken to be $1 \mathrm{GeV}$ as motivated in [22]. It has been demonstrated that this combination of matrix elements and parton showers gives a good description of the data in a wide range of the phase space specified by the cuts below [3, 20, 23, 24]. Nevertheless, there is some arbitrariness in the definition of the parton level. Investigations have shown that the determination of $\alpha_{s}$ can depend on the definition of the parton level at the order of a few per cent [25, 26]. The interpretation of these investigations is, however, difficult. It can be argued that this sensitivity is due to effects of missing higher order terms which may well be included in the systematic errors due to the uncertainties of the available QCD models, in the renormalisation scale and in the choice of the used jet algorithm (see below). Accordingly, no additional source of systematic error is included for this arbitrariness.

For the selected phase space region, in each $Q^{2}$ bin a correction factor to the experimental jet event rate is calculated using the LEPTO 6.5 generator from the ratio of the jet event rates obtained at the parton level after parton showering and at the detector level. In the same way also correction factors from the detector to the hadron level are determined. A possible model dependence introduced by this prescription is investigated by using the event generators HERWIG [27] and ARIADNE [28] and its effect is included in the systematic error.

The theoretical predictions are calculated using parton density parameterizations with the factorization scale $\mu_{f}^{2}$ given by $Q^{2}$. The parton densities are usually obtained via evolution from a low lying scale using a value $\alpha_{s}\left(M_{Z}^{2}\right)$ as input parameter, which may differ from that extracted in the analysis presented here. It will be shown that the error introduced by this inconsistency is small in the kinematic region considered.

\section{Phase Space Selection}

The events selected according to section 3 are subject to a jet classification applying the modified JADE jet algorithm as discussed in section \#. A resolution parameter of $y_{\text {cut }}=0.02$, in combination with the cut $W^{2}>5000 \mathrm{GeV}^{2}$ (section 3), implies an invariant mass squared of the two current jets which is greater than $100 \mathrm{GeV}^{2}$. After the jet classification a cut in the maximum jet angle $\left(\theta_{\max }=145^{\circ}\right)$ is applied to ensure that the jets are measured in the LAr calorimeter.

A cut at moderate values of $Q^{2}\left(Q^{2}>40 \mathrm{GeV}^{2}\right)$ ensures that $R_{2+1}$ is well described by LEPTO. In addition it avoids phase space regions where the variation of the QCD parameter $\Lambda$ is more important in the parton density functions than in the hard-scattering cross sections [29]. Furthermore, since this cut does not suppress parton showers sufficiently, additional requirements 
are necessary. Parton showers produce jets with predominantly small angles $\left(\theta_{j e t}\right)$ relative to the incident proton direction and small values of

$$
z_{p}=\min _{i=1,2} \frac{E_{j e t, i}\left(1-\cos \theta_{j e t, i}\right)}{\sum_{j=1}^{2} E_{j e t, j}\left(1-\cos \theta_{j e t, j}\right)} .
$$

The values of $z_{p}$ determined by the measured energies $E_{j e t, j}$ and angles $\theta_{j e t, j}(j=1,2)$ of the jets in the laboratory frame are in the range $0<z_{p}<0.5$. The $\theta_{j e t}$ and $z_{p}$ distributions are strongly correlated but are projections of different regions of the jet phase space in the $\left(\theta_{j e t}, z_{p}\right)$-plane. Both are used in this analysis to restrict the jet acceptance region.

In fig. 3a the distribution of the smallest jet angle $\left(\theta_{\text {jet,min }}\right)$ relative to the incident proton direction in a $(2+1)$ jet event using the JADE algorithm is plotted for $Q^{2}>40 \mathrm{GeV}^{2}$ and $z_{p}>0.1$. The curves are normalized to the number of events. It can be seen that above $\theta_{\text {jet,min }}=10^{\circ}$ the data are well reproduced by the MEPS model after detector simulation. In addition the MEPS model at the parton level and the MEPJET calculation (with MRSH as parton density parameterization and $\Lambda \frac{(4)}{M S}=230 \mathrm{MeV}$ ) agree reasonably well with each other. Both the MEPS model and the NLO calculation predict however fewer $(2+1)$ jet events with smallest $\theta_{j e t, m i n}$ values than measured in the data. A $\theta_{j e t}$ cut suppresses parton showers and hadronization effects efficiently. The $z_{p}$ distribution is shown for $Q^{2}>40 \mathrm{GeV}^{2}$ and $\theta_{\text {jet }}>10^{\circ}$ in fig. $3 \mathrm{~b}$ b. The final phase space selection fulfilling the criteria described in section 5 is therefore given by the requirements $Q^{2}>40 \mathrm{GeV}^{2}, \theta_{\text {jet }}>10^{\circ}$ and $z_{p}>0.1$, and is hereafter referred to as the central cut scenario. With these requirements the final event sample contains $1038(2+1)$ jet events of which 689 have $Q^{2}>100 \mathrm{GeV}^{2}$.

Due to effects of the finite resolution of energy and angles in the calculation of $m_{i j}^{2}$, there are migrations between jet classes at the levels of the detector, hadrons and partons. The efficiency
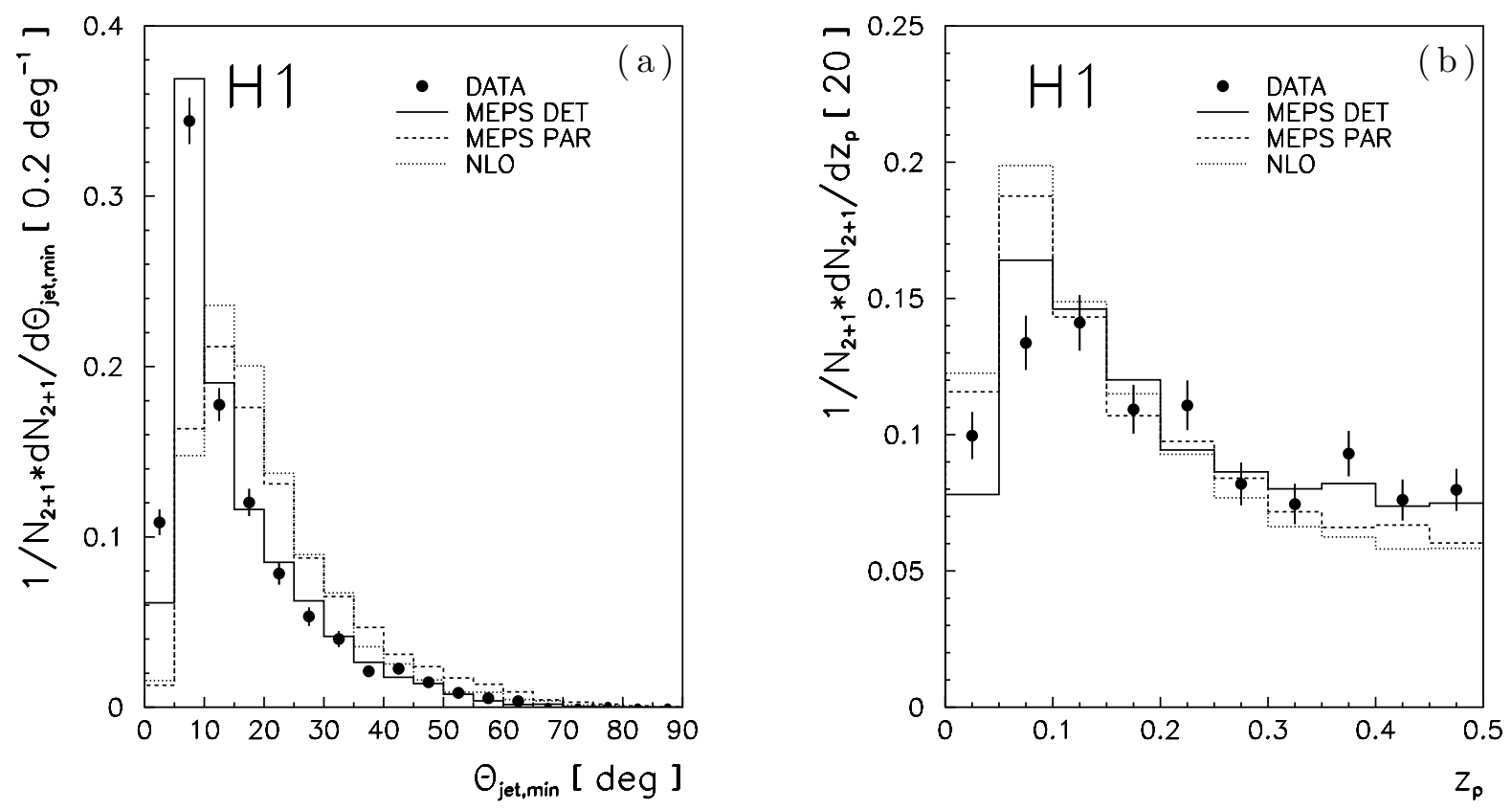

Figure 3: a) The $\theta_{\text {jet,min }}$ distribution of the (2+1) jet events for $Q^{2}>40 \mathrm{GeV}^{2}, z_{p}>0.1$ and $\theta_{\max }=145^{\circ}$; b) The $z_{p}$ distribution of the $(2+1)$ jet events for $Q^{2}>40 G e V^{2}$, $10^{\circ}<\theta_{\text {jet }}<145^{\circ}$. The JADE algorithm is used to define the jets; in both figures the data are represented by the points with statistical error bars only. The solid line shows the prediction of the MEPS model at the detector level and the dashed line the prediction of the same model at the parton level. The NLO calculation $\left(\Lambda \frac{(4)}{M S}=230 \mathrm{MeV}\right)$ is given by the dotted line. All curves are normalized to the number of $(2+1)$ jet events. 

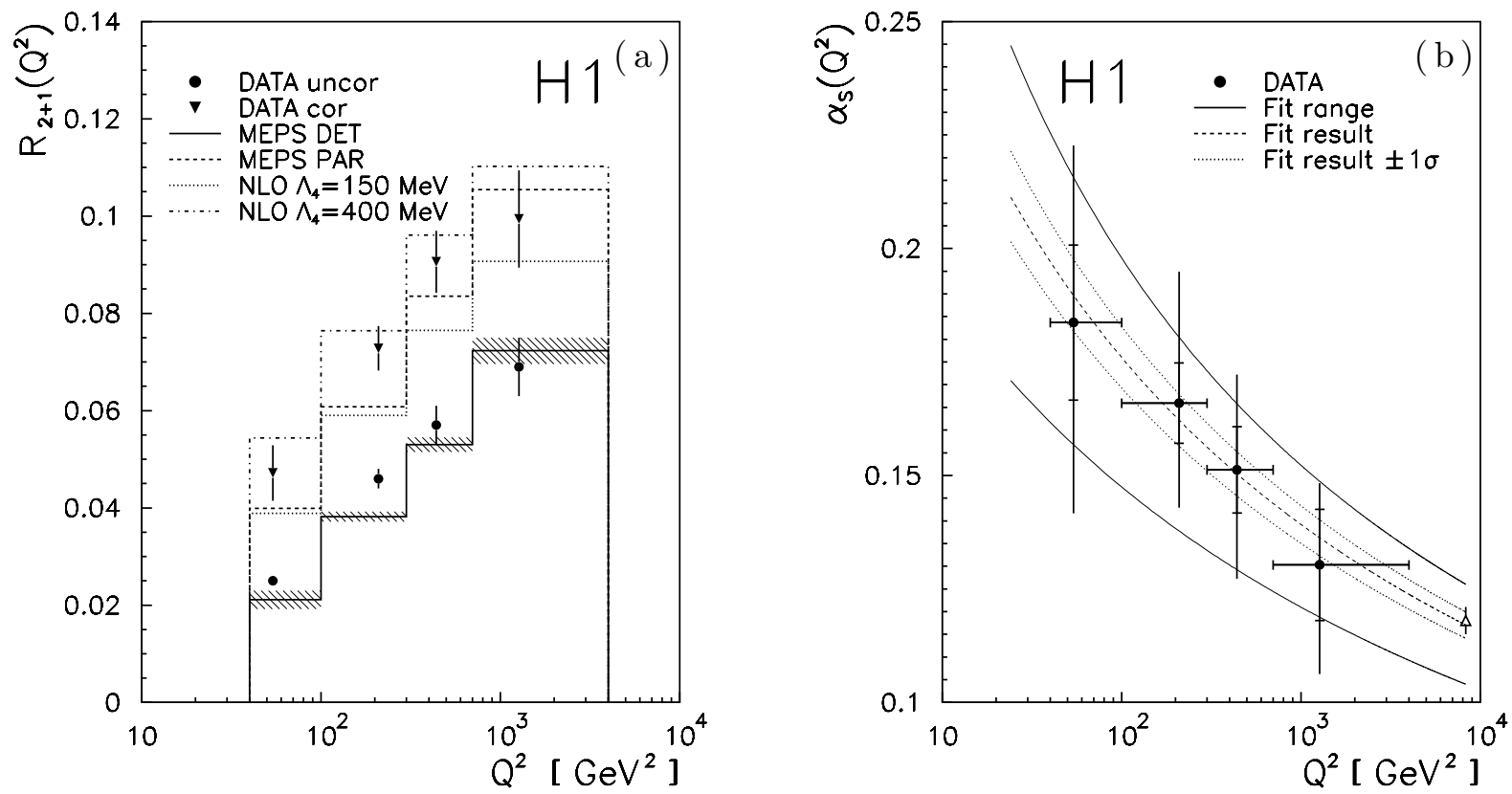

Figure 4: The $\alpha_{s}$ determination at four values of $Q^{2}$ and the evolution to the scale $Q^{2}=M_{Z}^{2}$ using the modified JADE jet algorithm. The data points are placed at the average $Q^{2}$ in each bin.

a) The measured $R_{2+1}\left(Q^{2}\right)$ (points) and the jet event rates corrected to the parton level (triangles) together with the prediction of the MEPS Monte Carlo at the detector level including statistical error bands (solid line), parton level (dashed line) and the MEPJET NLO calculations (dotted and dashed dotted lines) for two values of $\Lambda \frac{(4)}{M S}$. The event rates are determined for $y_{\text {cut }}=0.02, Q^{2}>40 \mathrm{GeV}^{2}, \theta_{\max }=145^{\circ}, \theta_{\text {jet }}>10^{\circ}$ and $z_{p}>0.1$. The predictions are based on the MRSH parton density parameterization.

b) The $\alpha_{s}$ values derived from the jet event rates as function of $Q^{2}$ with statistical errors from the data and the correction factors as inner error bars, and total errors as full error bars. The width of the $Q^{2}$ bins are indicated by the horizontal bars. The fit result (dashed line) and the 1 s.d. errors (dotted lines) are based on the individual $\alpha_{s}\left(Q^{2}\right)$ values with their statistical errors only. The solid lines represent the obtained range for $\alpha_{s}\left(M_{Z}^{2}\right)$ taking into account the systematic uncertainties, except the jet algorithm error. The open triangle at $Q^{2}=M_{Z}^{2}$ indicates the world average $\alpha_{s}\left(M_{Z}^{2}\right)=0.118 \pm 0.003$ [1]

and purity of the selected BEMC (LAr) sample are $27 \%$ (40\%) and $57 \%$ (69\%) with respect to the jet classification at the parton level and the above cuts [23].

The influence of radiative QED corrections on the $(2+1)$ jet event rate is studied with the event generator DJANGO [30] and is negligible. In this acceptance region the NLO corrections to the $\mathrm{LO}$ calculation are less than $30 \%$ in the investigated range of $Q^{2}$ and the Bjorken variable $x_{B}$.

Using the JADE algorithm the measured $(2+1)$ jet event rate $R_{2+1}$ for $Q^{2}>40 \mathrm{GeV}^{2}$, $\theta_{\text {jet }}>10^{\circ}, z_{p}>0.1$ is shown in fig. Ta. The data above $Q^{2}=40 \mathrm{GeV}^{2}$ are divided into four bins containing approximatively equal numbers of $(2+1)$ jet events. The measurements compare reasonably with the MEPS Monte Carlo at the detector level. In fig. Ha it can also be seen that the jet event rate at the parton level in the MEPS model lies well between the MEPJET prediction for $\Lambda \frac{(4)}{M S}=150 \mathrm{MeV}$ and $\Lambda \frac{(4)}{M S}=400 \mathrm{MeV}$. 


\begin{tabular}{|c|c|c|c|c|c|}
\hline$Q^{2}\left[\mathrm{GeV}^{2}\right]$ & $R_{2+1}\left[10^{-3}\right]$ & $R_{2+1}^{\text {had }}\left[10^{-3}\right]$ & $R_{2+1}^{\text {par }}\left[10^{-3}\right]$ & $f($ det $\rightarrow$ had $)$ & $f($ det $\rightarrow$ par $)$ \\
\hline \hline $40-100$ & $25 \pm 1_{-2}^{+2}$ & $33 \pm 4_{-3}^{+4}$ & $47 \pm 6_{-9}^{+4}$ & $1.32 \pm 0.15+0.14$ & $1.88 \pm 0.21-0.03$ \\
\hline $100-300$ & $46 \pm 2_{-4}^{+3}$ & $60 \pm 4_{-5}^{+4}$ & $73 \pm 5_{-8}^{+9}$ & $1.30 \pm 0.04+0.00$ & $1.59 \pm 0.05-0.12$ \\
\hline $300-700$ & $57 \pm 4_{-0}^{+3}$ & $72 \pm 5_{-1}^{+4}$ & $91 \pm 6_{-13}^{+5}$ & $1.26 \pm 0.04-0.02$ & $1.60 \pm 0.05-0.14$ \\
\hline $700-4000$ & $69 \pm 6_{-1}^{+4}$ & $82 \pm 8_{-2}^{+5}$ & $99 \pm 10_{-11}^{+8}$ & $1.19 \pm 0.06-0.02$ & $1.43 \pm 0.07-0.09$ \\
\hline
\end{tabular}

Table 1: The measured $(2+1)$ jet event rates $\left(R_{2+1}\right)$ with statistical and systematic errors in different bins of $Q^{2}$ for the central cut scenario using the modified JADE algorithm and corrected to the hadron $\left(R_{2+1}^{\text {had }}\right)$ and parton level $\left(R_{2+1}^{\text {par }}\right)$. In addition the correction factors from the detector to the hadron $(f($ det $\rightarrow$ had $))$ and parton $(f($ det $\rightarrow$ par $))$ level are included with errors due to Monte Carlo statistics and the model uncertainty estimated by ARIADNE. The statistical errors on the measured $R_{2+1}$ are given by the data only, whereas the errors on the corrected (2+1) jet event rates $\left(R_{2+1}^{\text {had }}, R_{2+1}^{p a r}\right)$ include also the statistical errors from the Monte Carlo. The systematic errors on $R_{2+1}$ correspond to the uncertainty on the hadronic energy scale. The systematic errors on $R_{2+1}^{\text {had }}$ take into account the hadronic energy scale and model uncertainty. For $R_{2+1}^{p a r}$ also the effects of parton showers, hadronization parameters and $Q C D$ Monte Carlo models were taken into account similar to the error estimation on $\alpha_{s}$ as described later in the text.

\section{Determination of the Strong Coupling Constant}

The correction factors $f($ det $\rightarrow$ par $)$, necessary to obtain from the measured jet event rate $R_{2+1}$ the rate at the parton level $\left(R_{2+1}^{p a r}\right)$, are given in table 1 for the different $Q^{2}$ bins shown in fig. 国a. They are between 1.4 and 1.9. Also the factors $f(\operatorname{det} \rightarrow$ had $)$ for correcting the measured event rates to the hadron level are included in table1. It can be seen that hadronization and detector effects lead to lower observable $(2+1)$ jet event rates than at the parton level due to resolution effects. The statistical errors on the corrected event rates in table 1 include the statistical error from the data as well as from the calculation of the correction factors using the Monte Carlo generator. For all calculations the parton density distributions were parameterized using MRSH [32].

The experimental event rates corrected to the parton level $R_{2+1}^{p a r}$ are also plotted in fig. 4a for the standard set of cut parameters (see above) using the JADE algorithm. They are converted into four values of $\alpha_{s}\left(Q^{2}\right)$. The QCD parameter $\Lambda_{\frac{(4)}{M S}}$ in the 2-loop solution of the renormalization group equation is fitted [33] to these extracted values of $\alpha_{s}\left(Q^{2}\right)$ taking into account their statistical errors only. The results, together with the \pm 1 s.d. parameter error of the fit, are shown in figure बb. The observed $Q^{2}$ dependence is compatible with the expected theoretical evolution. The numerical result at $Q^{2}=M_{Z}^{2}$ is $\alpha_{s}\left(M_{Z}^{2}\right)=0.117 \pm 0.003$ (stat).

To obtain the systematic uncertainty on $\alpha_{s}\left(M_{Z}^{2}\right)$ the fit is repeated for various other scenarios changing cuts and parameters relevant for the jet analysis. This procedure automatically takes into account the correlation between the four bins in $Q^{2}$. All systematic errors discussed in detail below are summarized in table 2 .

In order to determine the systematic experimental error the following kinematic cuts are investigated:

- The cut in $z_{p}$ is varied between 0.05 and 0.15 for $\theta_{j e t}>5^{\circ}, 10^{\circ}, 15^{\circ}$ leaving the other parameters at their central values. In addition $\theta_{\max }$ is varied between $140^{\circ}$ and $150^{\circ}$ leaving the other parameters at their central values. The resulting error on $\alpha_{s}\left(M_{Z}^{2}\right)$ as determined from the maximum spread of all fit results is +0.004 and -0.004 . 
- The hadronic energy scale of the LAr calorimeter in the data reconstruction is varied by $\pm 4 \%$. The resulting error on $\alpha_{s}\left(M_{Z}^{2}\right)$ is +0.005 and -0.003 .

The systematic experimental error $(+0.006$ and -0.005$)$ on $\alpha_{s}\left(M_{Z}^{2}\right)$ is taken as the quadratic sum of the two errors given above. Effects due to the uncertainty of the energy of the scattered positron and the variation of kinematic cuts defined in section 3 are negligible.

A further source of systematic uncertainty stems from the dependence of the correction factors on the Monte Carlo models used to calculate them. These uncertainties are analyzed employing the following procedure:

- The LEPTO Monte Carlo uses the JETSET [34] fragmentation model. Several sets of parameters describing the hadronization and fragmentation as fitted by the LEP experiments [35] are used to study the stability of the correction factors from the hadron to the parton level. The widths of the Gaussian distributions for the transverse momenta of partons in the proton $\left(k_{T}(\operatorname{PARL}(14))\right.$ and $\left.\sigma_{p t}(\operatorname{PARL}(3))\right)$ are varied between $350-$ $700 \mathrm{MeV}$ and $440-700 \mathrm{MeV}$, respectively; the fragmentation parameter $a$ of the LUND fragmentation functions is varied between 0.1 and 1 ; the width $\sigma(\operatorname{PARJ}(21))$ in the gaussian $p_{x}$ and $p_{y}$ transverse momentum distributions for primary hadrons is changed from the default value $(360 \mathrm{MeV})$ to 250 and $450 \mathrm{MeV}$. In addition the momentum scale which determines the termination of the parton shower $\left(Q_{0}\right.$-parameters $\mathrm{PYPA}(22)$ and PARJ(82) in LEPTO for the initial and the final state partons respectively) are varied between 1 and $2 \mathrm{GeV}$ and between 1 and $4 \mathrm{GeV}$, respectively. Finally the lowest value of the invariant mass squared $\hat{s}$ of the two generated partons, for which the LO ME calculations are carried out, is changed from $4 \mathrm{GeV}^{2}$ to $25 \mathrm{GeV}^{2}$. The resulting fitted $\alpha_{s}\left(M_{Z}^{2}\right)$ values are in the range 0.115 to 0.121 .

- Instead of LEPTO, the HERWIG [27] Monte Carlo model with its different treatment of parton showering and hadronization is applied to calculate the $(2+1)$ jet event rate on parton and hadron level. HERWIG is able to describe the shape of the measured $R_{2+1}\left(Q^{2}\right)$ distribution. Using the same corrections for detector effects as determined with the LEPTO generator, the fit leads to a reduction of $\alpha_{s}\left(M_{Z}^{2}\right)$ by 0.007 .

\begin{tabular}{|l|c|c|}
\hline Source & Variations & $\Delta \alpha_{s}\left(M_{Z}^{2}\right)$ \\
\hline \hline Statistics & & \pm 0.003 \\
\hline \hline$\theta_{\text {jet }}$ and $z_{p}$ cut & variation in $\theta_{\text {jet }}$ and $z_{p}$, see text & ${ }_{-0.004}^{+0.004}$ \\
\hline Hadronic energy scale & $\pm 4 \%$ & ${ }_{-0.003}^{+0.005}$ \\
\hline \hline \multirow{3}{*}{ Model dependence } & LEPTO 6.5 & ${ }_{-0.002}^{+0.004}$ \\
\cline { 2 - 3 } & HERWIG 5.9 & -0.007 \\
\cline { 2 - 3 } & ARIADNE 4.08 & -0.005 \\
\hline \hline Parton density functions, & MRSAp-201, GRV92, CTEQ-4A1 & ${ }_{-0.003}$ \\
$\Lambda$ & $0.15,0.40 \mathrm{GeV}$ & ${ }_{-0.002}$ \\
\hline Renormalization and factorization scale & $\mu_{i}^{2}=1 / 4,4 Q^{2} \mathrm{i}=\mathrm{f}, \mathrm{r}$ & ${ }_{-0.007}^{+0.003}$ \\
\hline$y_{\text {cut }}$ & $0.015,0.025$ & ${ }_{-0.003}^{+0.002}$ \\
\hline \hline jet algorithms & $\mathrm{E} 0$ & +0.000 \\
& $\mathrm{P}$ & +0.006 \\
\hline
\end{tabular}

Table 2: The determination of various systematic uncertainties. The central cut scenario and the MRSH parton density parameterization are used as reference. 
- The event generator ARIADNE 4.08 [28] is also used to study the model dependence. This Monte Carlo model treats the gluon emission in the framework of the colour dipole model [36] as an alternative to the parton showers in LEPTO. In the selected phase space ARIADNE describes the data well with exception of the $R_{2+1}\left(Q^{2}\right)$ distribution. The fitted $\alpha_{s}\left(M_{Z}^{2}\right)$ value using fully simulated ARIADNE events deviates by -0.005 from the quoted result obtained with LEPTO.

The dependence of $\alpha_{s}\left(M_{Z}^{2}\right)$ on the choice of the Monte Carlo model is estimated by the quadratic sum of all these uncertainties to be +0.004 and -0.009 .

Finally the theoretical uncertainty is investigated by changing various input conditions for the NLO calculations:

- The MRSH parton density function (PDF) used in MEPJET is replaced by the MRSAp201 [37], GRV92 [38], and the CTEQ-4A1 [39] parameterizations. They all use a similar $\Lambda \frac{(4)}{M S}$ value of about $230 \mathrm{MeV}$. The largest change in the fitted value of $\alpha_{s}\left(M_{Z}^{2}\right)$ is \pm 0.001 . As a particular point of interest [40, 29] the correlation of the measured value of $\alpha_{s}$ in this analysis with the value used while fitting the PDFs is investigated. This is done using the GRV92 PDFs which are available for various $\Lambda \frac{(4)}{M S}$ values 41] in the range $0.15-0.40 \mathrm{GeV}$. Although $\alpha_{s}\left(M_{Z}^{2}\right)$ used in the PDFs changes by up to 0.017 , the fitted values only differ by +0.003 and -0.002 . This large reduction is due to the fact that the value of $\alpha_{s}$ influences the scaling violation, whereas $R_{2+1}\left(Q^{2}\right)$ is directly proportional to the strong coupling constant. In table 2 both errors were combined to one.

- The factorization scale $\mu_{f}^{2}$ and the renormalization scale $\mu_{r}^{2}$ are varied independently between $Q^{2} / 4$ and $4 Q^{2}$. The effect on the measured value of $\alpha_{s}\left(M_{Z}^{2}\right)$ is +0.003 and -0.007 .

- The dependence on the chosen value of $y_{\text {cut }}$ was investigated by varying $y_{\text {cut }}$ between 0.015 and 0.025 in the NLO calculation, LEPTO simulation and for the data. Above 0.025 the number of $(2+1)$ jet events rapidly decreases but the results are still consistent within the larger statistical error. Below 0.015 the regime of very small jet masses opens up, making the fit procedure unstable. The resulting error on $\alpha_{s}\left(M_{Z}^{2}\right)$ is +0.002 and -0.003 .

The total theoretical uncertainty $(+0.005$ and -0.008$)$ on $\alpha_{s}\left(M_{Z}^{2}\right)$ is obtained summing these three errors in quadrature.

The result of the analysis using the JADE algorithm, where the total systematic uncertainty is taken as the quadratic sum of all uncertainties discussed above, is:

$$
\alpha_{s}\left(M_{Z}^{2}\right)=0.117 \pm 0.003(\text { stat })_{-0.013}^{+0.009}(\text { sys }) .
$$

The solid lines in figure $4 \mathrm{~b}$ indicate the full range of $\alpha_{s}\left(Q^{2}\right)$ obtained in this experiment using the JADE jet algorithm adding statistical and systematic errors in quadrature at the scale $Q^{2}=M_{Z}^{2}$. They correspond to $\alpha_{s}\left(M_{Z}^{2}\right)=0.104$ and 0.126 .

To obtain the systematic uncertainties on the four measured values of $\alpha_{s}\left(Q^{2}\right)$ using the JADE algorithm the following method is applied. Each of the used scenarios, except the variation of the renormalization scale, results in measured values of $\alpha_{s}\left(Q^{2}\right)$ for the four bins in $Q^{2}$. From the values of the different cut scenarios the systematic experimental and theoretical uncertainties, as well as the model dependence in each bin, are determined in the same way as discussed above for the uncertainties on $\alpha_{s}\left(M_{Z}^{2}\right)$. The total systematic uncertainty using the JADE algorithm is given by the quadratic sum of these three error contributions. The outer error bars of the $\alpha_{s}\left(Q^{2}\right)$ points in figure $\$ \mathrm{~b}$ represent the total uncertainties, which are calculated as the quadratic sum of statistical errors and total systematic uncertainties (see table 3). 


\begin{tabular}{|c||c|c|c|c||c||c|}
\hline \multicolumn{1}{|c||}{} & \multicolumn{4}{c||}{ JADE } & E0 & P \\
\hline \hline$Q^{2}\left[\mathrm{GeV}^{2}\right]$ & $\alpha_{s}\left(Q^{2}\right)$ & stat & sys & tot & $\alpha_{s}\left(Q^{2}\right)$ & $\alpha_{s}\left(Q^{2}\right)$ \\
\hline \hline 54 & 0.184 & \pm 0.017 & ${ }_{-0.038}^{+0.035}$ & ${ }_{-0.042}^{+0.039}$ & 0.189 & 0.206 \\
\hline 209 & 0.166 & \pm 0.009 & ${ }_{-0.021}^{+0.028}$ & ${ }_{-0.023}^{+0.029}$ & 0.166 & 0.177 \\
\hline 440 & 0.151 & \pm 0.010 & ${ }_{-0.022}^{+0.018}$ & ${ }_{-0.024}^{+0.021}$ & 0.151 & 0.167 \\
\hline 1272 & 0.130 & \pm 0.012 & ${ }_{-0.020}^{+0.012}$ & ${ }_{-0.024}^{+0.018}$ & 0.127 & 0.128 \\
\hline \hline$M_{Z}^{2}$ & 0.117 & \pm 0.003 & ${ }_{-0.013}^{+0.009}$ & ${ }_{-0.013}^{+0.009}$ & 0.117 & 0.123 \\
\hline
\end{tabular}

Table 3: The values of $\alpha_{s}\left(Q^{2}\right)$ together with their statistical, systematic and total uncertainties for the modified JADE algorithm at different values of $Q^{2}$, which are taken to be the average in each considered $Q^{2}$ range (see table 1$)$. In addition the extracted $\alpha_{s}\left(Q^{2}\right)$ values using the modified EO and $P$ algorithms are included.

To study the dependence of the determined $\alpha_{s}\left(M_{Z}^{2}\right)$ value on different jet algorithms the analysis is redone for the central cut scenario using the $\mathrm{E} 0$ and $\mathrm{P}$ variants of the JADE jet algorithm. The obtained $\alpha_{s}$ values in the different bins of $Q^{2}$ are given in table 3. The extracted value of $\alpha_{s}\left(M_{Z}^{2}\right)$ based on the E0 algorithm, $\alpha_{s}\left(M_{Z}^{2}\right)=0.117$, is, as expected from a theoretical point of view, very close to the value determined using the JADE algorithm, whereas for the $\mathrm{P}$ algorithm the fitted value is increased by +0.006 . The final result of this analysis is therefore

$$
\alpha_{s}\left(M_{Z}^{2}\right)=0.117 \pm 0.003(\text { stat })_{-0.013}^{+0.009}(\text { sys })+0.006(\text { jet algorithm })
$$

where the last error reflects a possible sensitivity to the choice of the jet algorithm used.

Another approach taken by the H1 Collaboration is to perform a quantitative pQCD analysis based on jet event rates differential [26] in the JADE jet resolution parameter. This method using a similar phase space region leads to $\alpha_{s}\left(M_{Z}^{2}\right)=0.118 \pm 0.002(\text { stat })_{-0.008}^{+0.007}(\text { sys })_{-0.006}^{+0.007}$ (theory), fully consistent in value and magnitude with the result of the present analysis.

The combined result from studies of the hadronic final state in the experiments at LEP and SLC [31] is $\alpha_{s}\left(M_{Z}^{2}\right)=0.122 \pm 0.007$. Here the error is totally dominated by theoretical and model dependencies associated with the same error sources as in the analysis presented in this paper.

\section{Summary}

Jet production in neutral current deep inelastic ep scattering at HERA is studied using the modified JADE jet algorithm. The strong coupling constant $\alpha_{s}$ is determined over a wide range of $Q^{2}$ by evaluating jet event rates corrected to the parton level assuming the hadron-parton relation as given by the event generator LEPTO. The $Q^{2}$ dependence of the determined $\alpha_{s}$ values is compatible with the theoretical prediction of the renormalization group equation. A fit of the QCD parameter $\Lambda \frac{(4)}{M S}$ leads to $\alpha_{s}\left(M_{Z}^{2}\right)=0.117 \pm 0.003(\text { stat })_{-0.013}^{+0.009}($ sys $)$. The systematic error is dominated by the uncertainty in the hadronic energy scale, the renormalisation scale dependence, and the dependence on the Monte Carlo model. Adding statistical and systematic errors in quadrature yields $\alpha_{s}\left(M_{Z}^{2}\right)=0.117_{-0.013}^{+0.009}$ consistent with the world average $\alpha_{s}\left(M_{Z}^{2}\right)=$ $0.118 \pm 0.003$ [31]. A dependence of the extracted $\alpha_{s}\left(M_{Z}^{2}\right)$ value on variations of the JADE algorithm is found. The largest change observed is +0.006 , leading to the final result:

$$
\alpha_{s}\left(M_{Z}^{2}\right)=0.117 \pm 0.003(\text { stat })_{-0.013}^{+0.009}(\text { sys })+0.006(\text { jet algorithm }) .
$$




\section{Acknowledgments}

We are grateful to the HERA machine group whose outstanding efforts have made and continue to make this experiment possible. We thank the engineers and technicians for their work in constructing and now maintaining the $\mathrm{H} 1$ detector, our funding agencies for financial support, the DESY technical staff for continual assistance, and the DESY directorate for the hospitality which they extend to the non-DESY members of the collaboration.

\section{References}

[1] JADE Collaboration, W. Bartel et al., Z. Phys. C33 (1986) 23.

[2] D. Graudenz and N. Magnussen, Proc. Workshop 'Physics at HERA', DESY, Hamburg, Vol. 1 (1991) 261, and references therein.

[3] H1 Collaboration, T. Ahmed et al., Phys. Lett. B346 (1995) 415.

[4] ZEUS Collaboration, M. Derrick et al., Phys. Lett. B363 (1995) 201.

[5] D. Graudenz, PROJET4.1, CERN-TH.7420/94.

[6] T. Brodkorb and E. Mirkes, DISJET manual, Univ. of Wisconsin, MAD/PH/821 (1994);

T. Brodkorb and E. Mirkes, Univ. of Wisconsin, MAD/PH/820 (1994).

[7] E. Mirkes and D. Zeppenfeld, Phys. Lett. B380 (1996) 205.

[8] S. Catani and M. Seymour, Nucl. Phys. B485 (1997) 291.

[9] H1 Collaboration, I. Abt et al., Nucl. Instr. and Meth. A386 (1997) 310-347 and Nucl. Instr. and Meth. A386 (1997) 348-396.

[10] H1 Calorimeter Group, B. Andrieu et al., Nucl. Instr. Meth. A336 (1993) 460.

[11] H1 Calorimeter Group, B. Andrieu et al., Nucl. Instr. Meth. A350 (1994) 57.

[12] H1 Calorimeter Group, B. Andrieu et al., Nucl. Instr. Meth. A336 (1993) 499.

[13] S. Bentvelsen et al., Proc. Workshop 'Physics at HERA', DESY, Hamburg, Vol. 1 (1991) 23; K.C. Hoeger, ibid., Vol. 1, 43.

[14] M. Flieser, PhD thesis (in German), TU Munich Fakultät für Physik, 1996.

[15] H1 Collaboration, I. Abt et al. Nucl. Instr. and Meth. A386 (1997) 348.

[16] H1 SPACAL Group, R.D. Appuhn et al., Nucl. Instr. Meth. A386 (1997) 397.

[17] H1 Collaboration, Technical Proposal for the Upgrade of the Backward Region of the H1 Detector, DESY internal report PRC-93/02.

[18] H1 Collaboration, S. Aid et al., Nucl. Phys. B439 (1995) 471.

[19] R. Nisius, PhD thesis, Aachen preprint PITHA 94/21 (1994).

[20] H1 Collaboration, I. Abt et al., Z. Phys. C61 (1994) 59.

[21] G. Ingelman, A. Edin and J. Rathsman, Comp. Phys. Comm. 101 (1997) 108. 
[22] V.A. Khoze, Proc. Symposium 'Lepton and photon interactions at high energies', Stanford (1989) 387-402.

[23] Ch. Niedzballa, PhD thesis (in German), Aachen preprint PITHA 97/36 (1997).

[24] F. Squinabol, PhD thesis (in French), Orsay preprint LAL 97-20 (1997).

[25] OPAL Collaboration, M.Z. Akrawy et al., Z. Phys. C49 (1991) 375.

[26] H1 Collaboration, C. Adloff et al., DESY preprint 98-075 (1998).

[27] B.R. Webber, HERWIG version 5.9, unpublished program manual; see also: HERWIG 5.4, Proc. Workshop 'Physics at HERA', DESY, Hamburg, Vol. 3 (1991) 1354.

[28] L. Lönnblad, ARIADNE version 4.08, Comput. Phys. Commun. 71 (1992) 15, and references therein.

[29] J. Chýla and J. Rameš, Proceedings of the workshop "Future Physics at HERA" at DESY, Hamburg 1996, Vol. 1, 529-532; PRA-HEP/96-01.

[30] G.A. Schuler and H. Spiesberger, DJANGO version 6.2, unpublished program manual; see also: DJANGO 1.0, Proc. Workshop 'Physics at HERA', DESY, Hamburg, Vol. 3 (1991) 1419.

[31] Particle Data Group, R.M. Barnett et al., Phys. Rev. D54 (1996) 1.

[32] A.D. Martin, W.J. Stirling and R.G. Roberts, Proc. of the Workshop on Quantum Field theory and Theoretical Aspects of High Energy Physics, eds. B. Geyer and E.M. Ilgenfritz, 11 (1993), Durham, DTP 93-86, and Rutherford, RAL 93-077.

[33] F. James and M. Ross, MINUIT program manual (version 94.1), CERN Program Library.

[34] T. Sjöstrand, PYTHIA 5.7 and JETSET 7.4, physics and manual, CERN-TH 7112-93.

[35] K. Hamacher and M. Weierstall, DELPHI 95-80 PHYS 515.

[36] B. Andersson, G. Gustafson and L. Lönnblad, Nucl. Phys. B339 (1990) 393.

[37] A.D. Martin, R.G. Roberts and W.J. Stirling, Phys. Lett. B356 (1995) 89.

[38] M. Glück, E. Reya and A. Vogt, Z. Phys. C53 (1992) 127.

[39] CTEQ Collaboration, H.L. Lai et al., Michigan State Univ., MSU-HEP-41024 (1994).

[40] B.R. Webber, Proceedings of ICHEP in Glasgow, 20-27 July 1994, Vol. 1, 213.

[41] A. Vogt, Phys. Lett. B354 (1995) 145. 